\title{
Erratum: Imaging spin diffusion in germanium at room temperature [Phys. Rev. B 96, 014403 (2017)]
}

\section{Zucchetti, F. Bottegoni, C. Vergnaud, F. Ciccacci, G. Isella, L. Ghirardini, M. Celebrano,} F. Rortais, A. Ferrari, A. Marty, M. Finazzi, and M. Jamet

(Received 25 July 2017; published 7 August 2017)

DOI: 10.1103/PhysRevB.96.059901

The $x$ axes of Fig. 3 report the wrong units. The correct units should be micrometers instead of millimeters.
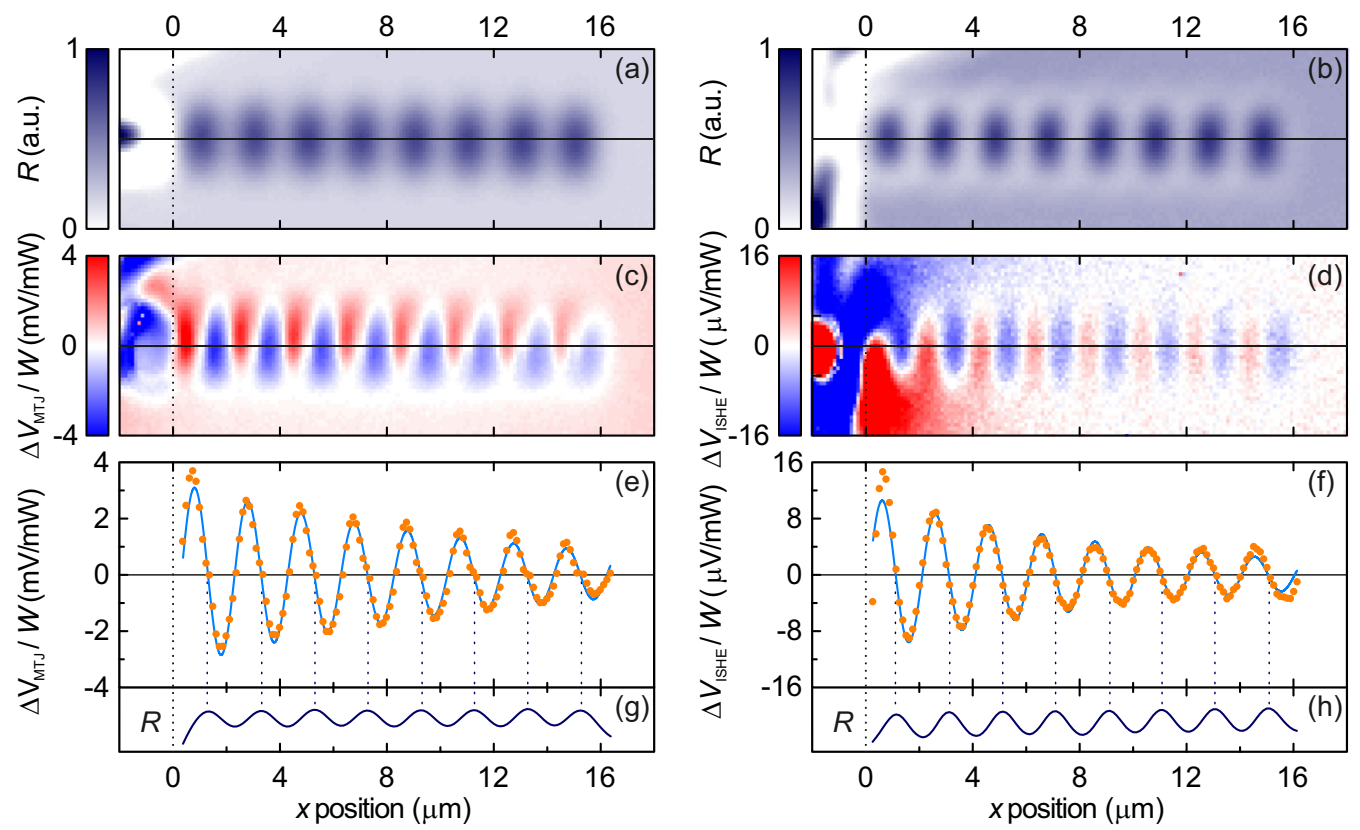

FIG. 3. (a) and (b) Reflectivity (R) optical images recorded on the magnetic tunnel junction (MTJ) and inverse spin Hall effect (ISHE) devices, respectively. (c) and (d) Corresponding simultaneously recorded MTJ and ISHE voltage signals. The incident power is $60 \mu \mathrm{W}$ for the MTJ sensor and $900 \mu \mathrm{W}$ for the ISHE detector. (e) and (f) Voltage profiles along $x$ across the centers of the Pt pads. In both cases, the detector is located at $x=0$. The dots are the experimental data, whereas the solid lines correspond to fits (see the text for the fitting function). The signals have been normalized to the laser power $W$. As illustrated by the dotted vertical line, corresponding to the maxima of the reflectivity $R$ profiles in $(\mathrm{g})$ and $(\mathrm{h})$, the signal is zero at the center of each Pt pad and positive (negative) on the left (right) edge. 